Bull. Austral. Math. Soc.

VoL. 53 (1996) [177-188]

\title{
NON DIFFERENTIABLE SYMMETRIC DUALITY
}

\section{Bertram Mond and Murray Schechter}

In this paper we construct dual pairs of problems, of both the Wolfe and MondWeir types, in which the objective contains a a support function and is therefore not differentiable. A special case which appears repeatedly in the literature is that in which the support function is the square root of a positive semidefinite quadratic form. This and other special cases can be readily generated from our result.

\section{INTRODUCTION}

In the literature of mathematical programming there are a large number of papers discussing duality theory for a problem involving the square root of a positive semidefinite quadratic function, $\sqrt{x^{T} B x}$. (See [2] and [3] and the references cited there). The popularity of this kind of problem seems to stem from the fact that, even though the objective function and/or constraint functions are nonsmooth, a simple representation for the dual problem may be found. Nonsmooth mathematical programming theory deals with much more general kinds of functions by means of generalised subdifferentials [1] or quasidifferentials [4]. However the square root of a positive semidefinite quadratic form is one of the few cases of a non-differentiable function for which one can write down the sub or quasi differentials explicitly. In this paper we replace $\sqrt{x^{T} B x}$ by a somewhat more general function, namely, the support function of a compact convex set, for which the subdifferential may be simply expressed. Using such a function we construct two different symmetric pairs of dual problems, one of the Wolfe type and one of the Mond-Weir type.

First we review some well known facts about support functions. Let $C$ be a compact convex set in $R^{n}$. The support function of $C$ is defined by

$$
s(x \mid C)=\max \left\{x^{T} y, y \in C\right\}
$$

Every sublinear (that is, positively homogeneous and subadditive) function defined on all of $R^{n}$ may be written as a support function and furthermore $C$ is uniquely determined by its support function. A support function, being convex and everywhere finite, has a subdifferential in the sense of convex analysis, that is, there exists $z$ such

Received 20th April, 1995

Copyright Clearance Centre, Inc. Serial-fee code: 0004-9729/96 SA2.00+0.00. 
that $s(y \mid C) \geqslant s(x \mid C)+z^{T}(y-x)$ for all $x$. The subdifferential of $s(x \mid C)$ (that is, the set of all such $z$ 's) is given by

$$
\partial s(x \mid C)=\left\{z \in C \text { such that } z^{T} x=s(x \mid C)\right\}
$$

We also require the concept of a normal cone. For any set $S$ the normal cone to $S$ at a point $x \in S$ is defined by

$$
N_{S}(x)=\left\{y \text { such that } y^{T}(z-x) \leqslant 0 \text { for all } z \in S\right\} .
$$

There is an easily verified relationship between normal cones and support functions of a compact convex set $\mathrm{C}$, namely, $y$ is in $N_{C}(x)$ if and only if $s(y \mid C)=x^{T} y$ or, equivalently, $x$ is in the subdifferential of $s$ at $y$.

Finally we shall need a form of the Fritz John conditions for a nonsmooth programming problem. The following is from [6]. Let $f$ and $g_{i}, i=1, \ldots m$ be subdifferentiable functions from $R^{n}$ into $R$ and let $C$ be a convex set in $R^{n}$. Consider the problem

$$
\begin{aligned}
\text { minimise } & f(x) \\
\text { subject to } & g_{i}(x) \leqslant 0, i=1, \ldots m \\
& x \in C .
\end{aligned}
$$

If $\bar{x}$ is an optimal solution to this problem then there exists a real number $\lambda$ and a vector $\mu \in R^{m}$, both $\geqslant 0$, such that

$$
\begin{gathered}
0 \in \lambda \partial f(\bar{x})+\sum_{i=i}^{m} \mu_{i} \partial g_{i}(\bar{x})+N_{C}(\bar{x}) \\
\lambda+\sum_{i=1}^{m} \mu_{i}>0 \\
\mu_{i} g_{i}(x)=0, i=1, \ldots m
\end{gathered}
$$

\section{A Wolfe Type Symmetric Dual Pair}

Consider the following pair of problems.

Problem (P)

Problem (D)

$$
\begin{array}{cc}
\text { minimise } & k(x, y)+s\left(x \mid C_{1}\right)-y^{T} \nabla_{2} k(x, y) \\
& \nabla_{2} k(x, y)-z \leqslant 0 \\
& x \geqslant 0, z \in C_{2} .
\end{array}
$$

$$
\begin{gathered}
\operatorname{maximise} \begin{array}{c}
k(u, v)-s\left(v \mid C_{2}\right)-u^{T} \nabla_{1} k(u, v) \\
\nabla_{1} k(u, v)+w \geqslant 0 \\
v \geqslant 0, w \in C_{1}
\end{array}
\end{gathered}
$$


Here $k$ is a differentiable mapping from $R^{n} \times R^{m}$ into $R$ and $C_{1}$ and $C_{2}$ are compact convex sets in $R^{n}$ and $R^{m}$ respectively.

Theorem 1. (Weak duality) Suppose that $k$ is convex in $x$ for fixed $y$ and is concave in $y$ for fixed $x$. Then $\inf (P) \geqslant \sup (D)$.

Proof: Suppose that $(x, y)$ and $(u, v)$ are feasible for problems (P) and (D) respectively. Then using the convexity and concavity properties of $k$ we have

$$
\begin{aligned}
& k(x, v)-k(x, y) \leqslant(v-y)^{T} \nabla_{2} k(x, y), \\
& k(x, v)-k(u, v) \geqslant(x-u)^{T} \nabla_{1} k(u, v) .
\end{aligned}
$$

Adding (-1) times equation (4) to equation (5) we get

$$
k(x, y)-k(u, v)-y^{T} \nabla_{2} k(x, y)+u^{T} \nabla_{1} k(u, v)+v^{T} \nabla_{2} k(x, y)-x^{T} \nabla_{1} k(u, v) \geqslant 0
$$

Using the constraints we get

$$
k(x, y)-k(u, v)-y^{T} \nabla_{2} k(x, y)+u^{T} \nabla_{1} k(u, v)+v^{T} z+x^{T} w \geqslant 0 .
$$

Finally, using $v^{T} z \leqslant s\left(v \mid C_{2}\right)$ and $x^{T} w \leqslant s\left(x \mid C_{1}\right)$ we get

$$
k(x, y)+s\left(x \mid C_{1}\right)-y^{T} \nabla_{2} k(x, y)-k(u, v)+s\left(v \mid C_{2}\right)+u^{T} \nabla_{1} k(u, v) \geqslant 0
$$

from which the result is immediate.

ThEOREM 2. (Strong duality) Suppose that $k$ is twice continuously differentiable and that $(\bar{x}, \bar{y}, \bar{z})$ is optimal for problem $(P)$. Suppose also that the Hessian $\left(\nabla_{2}\right)^{2} k(\bar{x}, \bar{y})$ is nonsingular. Then there exists $\alpha$ such that $(u, v, w)=(\bar{x}, \bar{y}, \alpha)$ is feasible for problem (D) and the objective function of problem (D) has the value $\min (P)$ at this feasible solution.

Proof: We recast problem $(P)$ into a form to which the Fritz John conditions stated in the introduction may be applied. Let $q=(x, y, z)$ and let

$$
\begin{aligned}
F(q) & =k(x, y)-y^{T} \nabla_{2} k(x, y)+s\left(q \mid C_{1} \times\{0\} \times\{0\}\right) \\
G(q) & =\nabla_{2} k(x, y)-z \\
H(q) & =-x \\
C & =R^{n} \times R^{n} \times C_{2} .
\end{aligned}
$$

Then problem $(\mathrm{P})$ can be restated as follows:

$$
\begin{aligned}
\text { minimise } & F(q) \\
& G(q) \leqslant 0 \\
& H(q) \leqslant 0 \\
& q \in C .
\end{aligned}
$$


In computing subdifferentials we shall use the following conventions: elements of $R^{n}$ will be written as columns and gradients also will be written as columns. $\nabla_{1} \nabla_{2} k(x, y)$ will be the matrix whose $j^{\prime}$ th column is the gradient with respect to $x$ of $\frac{\partial k}{\partial y_{j}}$.

Now we compute the desired subdifferentials.

$$
\partial F=\left[\begin{array}{c}
\nabla_{1} k(x, y)-\left(\nabla_{1} \nabla_{2} k(x, y)\right) y \\
-\left(\nabla_{2}^{2} k(x, y)\right) y \\
0
\end{array}\right]+\left[\begin{array}{l}
\alpha \\
0 \\
0
\end{array}\right]
$$

where $\alpha$ is any vector in $C$ satisfying $\alpha^{T_{x}}=s\left(x \mid C_{1}\right)$.

$$
\begin{aligned}
& G_{j}(q)= \frac{\partial k}{\partial y_{j}-z_{j}} \text { from which we get } \\
& \nabla_{q} G_{j}= {\left[\begin{array}{c}
\nabla_{x} \frac{\partial k}{\partial y_{j}} \\
\nabla_{y} \frac{\partial k}{\partial y_{j}} \\
-E_{j}
\end{array}\right] \text { (where } E_{j} \text { is column } j \text { of the identity matrix) } } \\
& H_{j}=-x_{j} \text { so } \nabla_{q} H_{j}=\left[\begin{array}{c}
-E_{j} \\
0 \\
0
\end{array}\right]
\end{aligned}
$$

Also note that $N_{C}(q)=\{0\} \times\{0\} \times N_{C_{2}}(z)$ at any $q \in C$. The Fritz John conditions (1) and (3) become

$$
\begin{gathered}
0 \in \lambda \partial F(\bar{q})+\sum \mu_{j} \partial G_{j}(\bar{q})+\sum \eta_{j} \partial H_{j}(\bar{q})+N_{C}(\bar{q}) \\
\sum \mu_{j} G_{j}(\bar{q})=\sum \eta_{j} H_{j}(\bar{q})=0
\end{gathered}
$$

Using the above formulas for subdifferentials we get (suppressing arguments)

$$
\begin{gathered}
\lambda\left[\nabla_{1} k-\left(\nabla_{1} \nabla_{2} k\right) \bar{y}+\alpha\right]+\left(\nabla_{1} \nabla_{2} k\right) \mu-\eta=0 \\
-\lambda\left(\nabla_{2}^{2} k\right) \bar{y}+\left(\nabla_{2}^{2} k\right) \mu=0 \\
-\mu \in-N_{C_{2}} \\
\alpha \in C_{1} \text { and } \alpha^{T} \bar{x}=s\left(\bar{x} \mid C_{1}\right) \\
\mu \geqslant 0 \text { and, for all } i, \mu_{i}\left(\frac{\partial k}{\partial y_{i}}-\bar{z}_{i}\right)=0 \\
\eta \geqslant 0 \text { and } \eta^{T} \bar{x}=0 .
\end{gathered}
$$

Suppose $\lambda=0$. From equation (7) and the nonsingularity of $\left(\nabla_{2}\right)^{2} k$ we deduce $\mu=0$ and from equation (6) we conclude that $\eta=0$. This is impossible since $\lambda, \eta$ and $\mu$ 
cannot all vanish, so $\lambda \neq 0$. We may and do assume that $\lambda=1$. Then equation (7) implies $\mu=\bar{y}$ and equation (6) simplifies to

$$
\nabla_{1} k+\alpha-\eta=0
$$

so that $\nabla_{1} k+\alpha \geqslant 0$. Then $(u, v, w)=(\bar{x}, \bar{y}, \alpha)$ is feasible for problem (D). Now we want to show that the objectives of the two problems have the same value. This is equivalent to the assertion that

$$
s\left(\bar{x} \mid C_{1}\right)+\bar{x}^{T} \nabla_{1} k(\bar{x}, \bar{y})=-s\left(\bar{y} \mid C_{2}\right)+\bar{y}^{T} \nabla_{2} k(\bar{x}, \bar{y}) .
$$

We shall show that both sides of equation (13) have the value 0 . By multiplying both sides of equation (12) by $\bar{x}^{T}$ we get from equations (9) and (11) that the left side vanishes. Using $\mu=\bar{y}$ equation (10) gives $\bar{y}^{T} \nabla_{2} k(\bar{x}, \bar{y})=\bar{y}^{T} \bar{z}$. But from $\bar{y} \in N_{C_{2}}(\bar{z})$ (equation (8)) we have $\bar{y}^{T} \bar{z}=s\left(\bar{y} \mid C_{2}\right)$, therefore $\bar{y}^{T} \nabla_{2} k(\bar{x}, \bar{y})=s\left(\bar{y} \mid C_{2}\right)$, so that the right side of equation (13) is zero as claimed.

Putting together the last two theorems and utilising the symmetry of the two problems we have the following:

COROLLARY 1. Suppose that $k$ is convex in its first argument and concave in its second argument and is twice continuousely differentiable with respect to all of its arguments. If problem $(P)$ has an optimal solution and $\left(\nabla_{2}\right)^{2} k$ is nonsingular there or if problem (D) has an optimal solution and $\left(\nabla_{1}\right)^{2} k$ is nonsingular there then both problems have optimal solutions and $\min (P)=\max (D)$.

Finally, we make the following observation: in the course of proving Theorem 2 we saw that $\bar{y} \geqslant 0$ (since $\bar{y}=\mu$ ). It follows from this that the set of optimal solutions of problem (P) remains unchanged if the condition $y \geqslant 0$ is added to the constraints. A corresponding statement may be made about problem (D). Also the weak duality theorem (Theorem 1 ) is clearly still valid if the conditions $y \geqslant 0$ and $u \geqslant 0$ are adjoined to problems (P) and (D) respectively. This observation leads to the following:

COROLlary 2. Suppose that conditions $y \geqslant 0$ and $u \geqslant 0$ are added to problems $(P)$ and $(D)$ respectively. Then Theorems 1 and 2 (weak and strong duality) and corollary 1 remain valid.

\section{A Mond-Weir Symmetric Dual Pair}

Here we present another symmetric dual pair involving a support function. In these problems gradients appear only in the constraints, as in all Mond-Weir duals. (See, for 
example, [5]).

Problem (P1)

Problem (D1)

$$
\begin{aligned}
\operatorname{minimise} & =k(x, y)+s\left(x \mid C_{1}\right)-y^{T} z \\
& \nabla_{2} k(x, y)-z \leqslant 0 \\
& y^{T}\left[\nabla_{2} k(x, y)-z\right] \geqslant 0 \\
& x \geqslant 0, z \in C_{2} .
\end{aligned}
$$

$$
\begin{aligned}
\operatorname{maximise} & =k(u, v)-s\left(v \mid C_{2}\right)+u^{T} w \\
& \nabla_{1} k(u, v)+w \geqslant 0 \\
& u^{T}\left[\nabla_{1} k(u, v)+w\right] \leqslant 0 \\
& v \geqslant 0, w \in C_{1} .
\end{aligned}
$$

It will prove convenient to have a name for the hypotheses we shall be using. Hypothesis (H1) Let $(\bar{x}, \bar{y}, \bar{z})$ and $(\bar{u}, \bar{v}, \bar{w})$ be feasible for problems (P1) and (D1) respectively. We assume that

1. $k(u, \bar{v})+u^{T} \bar{w}$ is a pseudoconvex function of $u$ at $u=\bar{u}$.

2. $k(\bar{x}, y)-y^{T} \bar{z}$ is a pseudoconcave function of $y$ at $y=\bar{y}$.

Note that Hypothesis (H1) is satisfied if $k(x, y)$ is convex in $x$ and concave in $y$, as is assumed in the duality theorem of the preceding section.

Theorem 3. (Weak Duality) Assume that hypothesis (H1) is satisfied. Then $\inf (P 1) \geqslant \sup (D 1)$.

Proof: Let $(x, y, z)$ and $(u, v, w)$ be feasible for problems (P1) and (D1) respectively. We shall show that

$$
k(x, y)+s\left(x \mid C_{1}\right)-y^{T} z \geqslant k(u, v)-s\left(v \mid C_{2}\right)+u^{T} w .
$$

By multiplying the first constraint of problem (D1) by $x^{T}$ and subtracting the second we get $(x-u)^{T}\left[\nabla_{1} k(u, v)-w\right] \geqslant 0$. From the first part of hypothesis (H1) we can conclude that

$$
k(x, v)+x^{T} w \geqslant k(u, v)+u^{T} w .
$$

In a similar fashion we get

$$
k(x, v)-v^{T} z \leqslant k(x, y)-y^{T} z
$$

From equations (14) and (15) we get

$$
k(x, y)+x^{T} w-y^{T} z \geqslant k(u, v)-v^{T} z+u^{T} w .
$$


Finally, note that $x^{T} w \leqslant s\left(x \mid C_{1}\right)$ and $v^{T} z \leqslant s\left(v \mid C_{2}\right)$. This together with equation (16) gives the desired result.

We now restate problem (P1) in a form which facilitates the application of the Fritz John conditions as stated in the introduction. Let $q=(x, y, z)$ and let

$$
\begin{aligned}
F(q) & =k(x, y)+s\left(q \mid\{0\} \times\{0\} \times C_{1}\right)-y^{T} z \\
G_{j}(q) & =\frac{\partial k(x, y)}{\partial y_{j}}-z_{j}, j=1 \ldots m \\
H_{j}(q) & =-x_{j}, j=1 \ldots n \\
K(q) & =-y^{T}\left[\nabla_{2} k(x, y)-z\right] \\
C & =R^{n} \times R^{m} \times C_{2} .
\end{aligned}
$$

Then problem (P1) may be restated as follows:

$$
\begin{aligned}
& \text { minimise } F(q) \\
& G \leqslant 0 \\
& H \leqslant 0 \\
& K \leqslant 0 \\
& q \in C .
\end{aligned}
$$

The Fritz John conditions then become

$$
\begin{gathered}
0 \in \lambda \partial F(\bar{q})+\sum \mu_{j} \partial G(\bar{q})+\sum \eta_{j} \partial H_{j}(\bar{q})+\omega K(\bar{q})+N_{C}(\bar{q}) \\
\mu_{j} G_{j}=\eta^{T} x=\omega K(\bar{q})=0 \\
\lambda, \omega \geqslant 0 \\
\mu \in R_{+}^{m}, \eta \in R_{+}^{n} .
\end{gathered}
$$

Computation of the required subdifferentials is similar to those done in section 2 and is omitted. The resulting conditions follow. It is to be understood that all functions are to be evaluated at $(x, \bar{y})$.

$$
\begin{gathered}
\lambda\left(\nabla_{1} k+\alpha\right)+\left(\nabla_{1} \nabla_{2} k\right)(\mu-\omega \bar{y})-\eta=0 \\
(\lambda-\omega)\left(\nabla_{2} k-\bar{z}\right)+\left(\nabla_{2}^{2} k\right)(\mu-\omega \bar{y})=0 \\
\lambda \bar{y}+(\mu-\omega \bar{y}) \in N_{C_{2}}(\bar{z}) \\
\left(\nabla_{2} k-\bar{z}\right)^{T} \mu=0 \\
\eta^{T} \bar{x}=0 \\
\omega \bar{y}^{T}\left(\nabla_{2} k-\bar{z}\right)=0 \\
\alpha \in C_{1}, \alpha^{T} \bar{x}=s\left(\bar{x} \mid C_{1}\right) .
\end{gathered}
$$


From equations (20) and (22) together we get

$$
(\mu-\omega \bar{y})^{T}\left(\nabla_{2} k-\bar{z}\right)=0,
$$

Multiplying equation (18) by $(\mu-\omega \bar{y})^{T}$ we get

$$
(\mu-\omega \bar{y})^{T}\left(\nabla_{2}^{2} k\right)(\mu-\omega \bar{y})=0 .
$$

We now assume that $\nabla_{2}^{2} k(\bar{x}, \bar{y})$ is either positive definite or negative definite. Then equation (25) gives

$$
\mu=\omega \bar{y}
$$

and then equation (18) becomes $(\lambda-\omega)\left(\nabla_{2} k-\bar{z}\right)=0$. We now assume further that $\nabla_{2} k(\bar{x}, \bar{y})-\bar{z} \neq 0$. Then we conclude from the last equation that

$$
\lambda=\omega
$$

From equation (26) we see that the middle term in equation (17) vanishes. From this we can deduce that $\lambda \neq 0$, for suppose $\lambda=0$. Then we get from equation (17) that $\eta=0$, from equation (27) that $\omega=0$ and from equation (20) that $\mu=0$. But this is impossible, since not all the multipliers vanish, hence $\lambda>0$. We can and do assume that $\lambda=1$. Then equation (17) tells us that $\nabla_{1} k(\bar{x}, \bar{y})+\alpha \geqslant 0$, so that $(u, v, w)=(\bar{x}, \bar{y}, \alpha)$ satisfies the first constraint of problem (D1). From equations (17) and (21) we see that $(\bar{x}, \bar{y}, \alpha)$ also satisfies the second constraint of problem (D1) (with $=$ as well as with $\leqslant$ ). From equation (26) we have $\bar{y}=\mu$ ( last constraint $w \in C$ is satisfied because of equation (23). Thus $(\bar{x}, \bar{y}, \alpha)$ is feasible for problem (D1). Furthermore, the objective functions of problems (P1) and (D1) have the same value when $(x, y, z)=(\bar{x}, \bar{y}, \bar{z})$ and $(u, v, w)=(\bar{x}, \bar{y}, \alpha)$. To verify this it has only to be shown that

$$
s\left(\bar{x} \mid C_{1}\right)-\bar{y}^{T} \bar{z}=-s\left(\bar{y} \mid C_{2}\right)+\bar{x}^{T} \alpha .
$$

Because of equation (23) this reduces to $\bar{y}^{T} \bar{z}=s\left(\bar{y} \mid C_{2}\right)$. This is an immediate consequence of the fact that $\bar{y} \in N_{C_{2}}(\bar{z})$ (equation (19)).

Combining what has just been proved with weak duality we get the following:

THEOREM 4. (Strong Duality) Suppose that $(\bar{x}, \bar{y}, \bar{z})$ is optimal for problem (P1), that hypothesis $(H 1)$ is satisfied, that $\nabla_{2}^{2} k(\bar{x}, \bar{y})$ is positive or negative definite and that $\nabla_{2} k(\bar{x}, \bar{y}) \neq \bar{z}$. Then there exists $\bar{w}$ such that $(u, v, w)=(\bar{x}, \bar{y}, \bar{w})$ is optimal for problem (D1) and furthermore $\min (P 1)=\max (D 1)$.

It is not clear that the hypothesis $\nabla_{2} k(\bar{x}, \bar{y})-\bar{z} \neq 0$ will usually be satisfied, since this rules out one way of satisfying the first and second constraints of problem (P1). We shall see below that one can expect that this condition will frequently be satisfied. 
By virtue of the symmetry of problems (P1) and (D1) an analogous statement may be made in which problem (D1) is assumed to have an optimal solution and and the conclusion is that problem (P1) has an optimal solution and $\max (\mathrm{D} 1)=\min (\mathrm{P} 1)$.

In the proof of the strong duality theorem it was noted that not only is $(\bar{x}, \bar{y}, \alpha)$ feasible for problem (D1) but the second constraint $u^{T}\left[\nabla_{1} k(u, v)+w\right] \geqslant 0$ is actually satisfied as an equality. The strong duality theorem then holds with problem (D1) changed to make this constraint an equality constraint. Suppose we make the corresponding constraint in problem (P1) an equality also. Clearly, weak duality is still valid. In the Fritz John conditions the multiplier $\omega$ would no longer be required to be non-negative. By examining the proof of the strong duality theorem we see that the condition $\omega \geqslant 0$ was never used, so the proof is valid even when the second constraint in each problem is changed into an equality. Also note that while $y$ is not required to be non-negative in problem (P1) we found that, at an optimal solution, it is. We sum up these observations in the following:

COROLLARY 3. Suppose that in problems (P1) and (D1) the second constraint is changed from an inequality to an equality. Then the optimal solutions are unchanged and the weak and strong duality theorems are still valid. Also if the conditions $y \geqslant 0$ and $u \geqslant 0$ are added to the constraints of problems (P1) and (D1) respectively the optimal solutions are unchanged and the weak and strong duality theorems are still valid.

We now consider the possibility of the hypothesis of Theorem 4 being satisfied. Hypothesis (H1), needed for weak duality requires that the function $k(\bar{x}, y)-y^{T} \bar{z}$ be pseuodoconcave in $y$, but it is required that $\nabla_{2}^{2} k(\bar{x}, \bar{y})$ be positive or negative definite for strong duality. The assumption that $\nabla_{2}^{2} k(\bar{x}, \bar{y})$ is negative definite on the entire feasible set will guarantee that both these conditions are satisfied (although it's much more than is needed). Let us make this assumption for now. Consider the hypothesis $\nabla_{2} k(\bar{x}, \bar{y})-\bar{z} \neq 0$. Let $f(y)=k(\bar{x}, y)-y^{T} \bar{z}$. Then the minimum of $f$ over all $y$ satisfying the first and second constraints of problem (P1) occurs at $y=\bar{y}$. Note that $\nabla f=\nabla_{2} k-z$. Suppose that, contrary to the hypothesis of Theorem 4, $\nabla_{2} k(\bar{x}, \bar{y})-\bar{z}=0$. Then $\bar{y}$ is a critical point of $f$. But since $f$ is strictly concave at $\bar{y}$, this means that $f$ has a maximum at $\bar{y}$. We may conclude that $\bar{y}$ is the only solution of the system

$$
\begin{array}{r}
\nabla_{2} k(\bar{x}, y)-\bar{z} \leqslant 0 \\
y^{T}\left[\nabla_{2} k(\bar{x}, y)-\bar{z}\right] \geqslant 0
\end{array}
$$

in some neighbourhood of $\bar{y}$. While it is possible that a system of inequalities has an isolated solution, this is not usually the case. This tells us that in most problems we 
can expect it to be the case that the remaining hypothesis, namely $\nabla_{2}(\bar{x}, \bar{y})-\bar{z} \neq 0$, is satisfied.

ExAmPLe. Let $n=m=1$, let $k(x, y)=x^{2}-y^{2}$ and let $C_{1}=C_{2}=[0,1]$. Then $s\left(x \mid C_{1}\right)=x_{+}=(x+|x|) / 2$. Problem (P1) becomes

Problem (P1.1)

$$
\begin{aligned}
\operatorname{minimise} & x^{2}-y^{2}+x_{+}-y z . \\
& -2 y-z \leqslant 0 \\
& y[-2 y-z] \geqslant 0 \\
& x \geqslant 0, z \in[0,1] .
\end{aligned}
$$

We can minimise over $x$ independently of $y$ and $z$ and we find that the minimum occurs at $\bar{x}=0$. To minimise over $(y, z)$ we first hold $z$ fixed in $[0,1]$. We find that, independently of $z$, the minimum accurs at $\bar{y}=0$ and the value of the objective function is 0 , therefore the optimal solution is $(0,0, \bar{z})$ where $\bar{z}$ can be any point in $[0,1]$. The condition $\nabla_{2} k(\bar{x}, \bar{y})-\bar{z} \neq 0$ is satisfied unless we are unlucky enough to choose $\bar{z}=0$. (Even if we did this, the conclusion of the strong duality theorem would still be valid).

\section{Special Cases}

As mentioned in the introduction, a frequently occurring example of a nondifferentiable support function is $\sqrt{x^{T} B x}$, where $B$ is a positive semidefinite matrix. It is readily shown that $\sqrt{x^{T} B x}=s\left(x \mid C_{1}\right)$ where $C_{1}=\left\{B w, w^{T} B w \leqslant 1\right\}$ and that this set $C_{1}$ is compact and convex. We briefly indicate what both the Wolfe symmetric dual pair and the Mond-Weir symmetric dual pair look like in these cases.

Let $C$ be another positive semidefinite matrix and let $C_{2}=\left\{C w, w^{T} C w \leqslant 1\right\}$. Then the dual pair, problems (P) and (D), becomes

Problem (P2)

$$
\begin{array}{cc}
\text { minimise } & k(x, y)+\sqrt{x^{T} B x}-y^{T} \nabla_{2} k(x, y) \\
& \nabla_{2} k(x, y)-C z \leqslant 0 \\
& z^{T} C z \leqslant 1 \\
& x \geqslant 0 .
\end{array}
$$

Problem (D2)

$$
\begin{array}{cc}
\operatorname{maximise} & k(u, v)-\sqrt{v^{T} C v}+u^{T} \nabla_{1} k(u, v) \\
& \nabla_{1} k(u, v)+B w \geqslant 0 \\
& w^{T} B w \leqslant 1 \\
v & \geqslant 0 .
\end{array}
$$


This is the pair studied in [3], except that Chandra and Husain add the constraints $y \geqslant 0$ and $u \geqslant 0$ to problems (P2) and (D2) respectively. As corollary 2 points out, this may be done without changing the duality theorems.

Using the same choices for $C_{1}$ and $C_{2}$ (P1) and (D1) become

Problem (P3)

$$
\begin{aligned}
\text { minimise } & k(x, y)+\sqrt{x^{T} B x}-y^{T} C z \\
& \nabla_{2} k(x, y)-C z \leqslant 0 \\
& y^{T}\left[\nabla_{2} k(x, y)-C z\right] \geqslant 0 \\
& z^{T} C z \leqslant 1 \\
& x \geqslant 0
\end{aligned}
$$

Problem (D3)

$$
\begin{aligned}
\operatorname{maximise} & k(u, v)-\sqrt{v^{T} C v}+u^{T} B w \\
& \nabla_{1} k(u, v)+B w \geqslant 0 \\
& u^{T}\left[\nabla_{1} k(u, v)+B w\right] \leqslant 0 \\
& w^{T} B w \leqslant 1 \\
& v \geqslant 0
\end{aligned}
$$

This is precisely the pair studied in [2], where the strong and weak duality theorems were proved. Note also that three other dual pairs can be generated from these problems by use of Corollary 3 .

\section{ReFERENCES}

[1] F.H. Clarke, Optimization and nonsmooth analysis (Wiley, New York, 1983).

[2] S. Chandra, B.D. Craven and B. Mond, 'Generalized concavity and duality with a square root term', in Mathematische Operationsforschung und Statistic Series optimization 16, 1975, pp. 653-662.

[3] S. Chandra and I. Husain, 'Symmetric dual nondifferentiable programs', Bull. Autral. Math. Soc. 24 (1981), 295-307.

[4] V.F. Demyanov and L.C.W. Dixon, Quasidifferential calculus, Math. Prog. Study 29 (North-Holland, Amsterdam, 1986).

[5] B. Mond and T. Weir, 'Generalized concavity and duality', in Generalized concavity in optimization and economics, (S. Schiable and W.T. Ziemba, Editors) (Academic Press, New York, 1981).

[6] M. Schechter, 'More on subgradient duality', J. Math. Anal. Appl. 71 (1979), 251-262. 
Department of Mathematics La Trobe University

Bundoora Vic $\mathbf{3 0 8 3}$

Australia

and

Department of Mathematics

Lehigh University

Bethlehem PA 18105

United States of America
Department of Mathematics

Lehigh University

Bethlehem PA 18105

United States of America 